\title{
Amaryllidaceae del Tolima: importancia para el tratamiento de la enfermedad de Alzheimer
}

\section{Amaryllidaceae from Tolima: the importance for the treatment of Alzheimer's disease}

\author{
Adriana Lucía Oliveros Lozada ${ }^{1}$
}

()

Recepción: 26/09/2020
-

Aprobación: 04/11/2020
()

Publicación: 18/12/2020

Para citar este artículo:

Oliveros Lozada, A. L. (2020). Amaryllidaceae del Tolima: importancia para el tratamiento de la enfermedad de Alzheimer. Indagare, (8), 254-259.

https://doi.org/10.35707/indagare/824

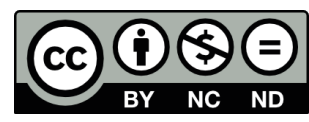

${ }^{1}$ Grupo de investigación Naturatu, Universidad de Ibagué, Colombia. ORCID: 0000-0002-7574-873X. Correo electrónico: adriana.oliveros@unibague.edu.co 


\title{
Resumen
}

El proyecto de investigación regional Amaryllidaceae del Tolima: fuente inexplorada de alcaloides para el tratamiento de la enfermedad de Alzheimer es dirigido por la doctora Natalie Charlotte Cortés Rendón, docente de la Facultad de Ciencias Naturales y Matemáticas. La investigadora habla sobre la importancia de la familia Amaryllidaceae y sus alcaloides para el tratamiento de afecciones neurodegenerativas como la enfermedad de Alzheimer y en otros blancos moleculares potenciales para esta familia botánica, como el cáncer.

\begin{abstract}
The regional research project Amaryllidaceae from Tolima: an unexplored source of alkaloids for the treatment of Alzheimer's disease is led by Dr. Natalie Charlotte Cortés Rendón, professor at the Faculty of Natural Sciences and Mathematics. The researcher addresses the importance of the Amaryllidaceae family and its alkaloids for the treatment of neurodegenerative conditions such as Alzheimer's disease and other potential molecular targets for this botanical family, like cancer.
\end{abstract}

\footnotetext{
(A) Palabras claves: Amaryllidaceae, alcaloide, afectaciones neurodegenerativas, enfermedad de Alzheimer, cáncer, galantamina. Key words: Amaryllidaceae, alkaloid, neurodegenerative disorders, Alzheimer's disease, cancer, galantamine.
}

La familia Amaryllidaceae es una familia de plantas perennes o caducifolias con bulbos tunicados, esporádicamente con rizomas (Alzate, Lesmes, Cortés, Varela \& Osorio, 2019) y se distribuye en zonas tropicales de Sur América y África del Sur (Meerow \& Snijman, 1998). En Colombia, la familia Amaryllidaceae está representada por 18 géneros y 48 especies (Alzate et al., 2019), entre nativas, endémicas y algunas introducidas. En ciertas especies de la familia Amaryllidaceae se puede encontrar el alcaloide galantamina, medicamento aprobado por la U.S Food and Drug Administration (FDA) para el tratamiento sintomático de la enfermedad de Alzheimer (Cabezas et al., 2013).

El proyecto Amaryllidaceae del Tolima: fuente inexplorada de alcaloides para el tratamiento de la enfermedad de Alzheimer tiene como objetivo investigar el potencial farmacológico de los alcaloides de la familia Amaryllidaceae que se encuentra en el departamento del Tolima para el tratamiento de la enfermedad de Alzheimer. Este 
proyecto es liderado por Natalie Charlotte Cortés Rendón, profesional en Química, magíster en Ciencias Farmacéuticas y Alimentarias y doctora en Ciencias Farmacéuticas y Alimentarias, estudios desarrollados en la Universidad de Antioquia. Actualmente está vinculada a la Universidad de Ibagué como docente de tiempo completo de la Facultad de Ciencias Naturales y Matemáticas, además es investigadora de los grupos de investigación Naturatu y QBOSMO.

La doctora Cortés tiene nueve años de experiencia en investigación científica, es autora y coautora de varios artículos científicos. Asimismo, se destaca el capítulo de libro titulado Amaryllidaceae alkaloids and neuronal cell protection publicado en el libro Pathology (Cortés et al., 2020). En su experiencia profesional se ha desempeñado como docente de la Universidad de Antioquia, la Institución Universitaria Colegio Mayor de Antioquia y la Universidad de Ibagué.

Figura 1. Retrato de Natalie Charlotte Cortés Rendón

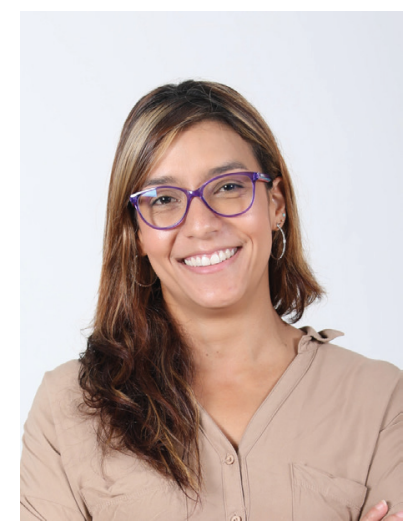

Fuente: Comunicación institucional, Universidad de Ibagué

\section{¿Cómo inicia su proceso como investigadora científica?}

Mi proceso empieza cuando inicié mi pregrado en Química en el año 2003, y desde siempre el enfoque de la carrera fue dirigido hacia la investigación. Luego, en el año 2011 comencé mi maestría en la Facultad de Ciencias Farmacéuticas y Alimentarias en la Universidad de Antioquia, donde continué con mi formación científica, ahora en química aplicada en el campo de las Ciencias Farmacéuticas. Allí adelanté investigaciones sobre la familia Amaryllidaceae y su actividad biológica para el tratamiento sintomático de la enfermedad de Alzheimer y profundicé aún más con mis estudios de doctorado en este mismo tema. 


\section{¿Cuál es su línea de investigación?}

Mi línea de investigación es Actividad biológica de compuestos de origen natural, dado que además de evaluar la actividad neuroprotectora, también estudio el potencial antioxidante de los compuestos extraídos de fuentes naturales.

¿En qué consiste el proyecto de investigación Amaryllidaceae del Tolima: fuente inexplorada de alcaloides para el tratamiento de la enfermedad de Alzheimer?

Para contestar esa pregunta, voy a hablar un poco de la historia de por qué esa familia y por qué el enfoque en la enfermedad de Alzheimer. Como comenté antes, mi maestría y doctorado los desarrollé con una familia botánica llamada Amaryllidaceae. Esa familia se encuentra ampliamente distribuida en todo el mundo y Colombia no es la excepción. En nuestro país contamos con una importante variedad de especies, pero con todo el trabajo que he desarrollado en maestría y doctorado nunca evalué Amaryllidaceae del Tolima. Aunque Tolima es muy diverso en flora y fauna, existe poca información reportada de estas plantas en el departamento. Debido a esto decidí que esta región sería el foco geográfico del estudio, porque gracias a las condiciones edafoclimáticas que se tienen hay un potencial químico y biológico aún desconocido.

¿Porquéen Alzheimer? Estas plantas producenun tipo especial dealcaloides, llamados alcaloides de Amaryllidaceae. Entre ellos, hay uno que es un medicamento aprobado por la $\mathrm{FDA}^{2}$ para el tratamiento sintomático de la enfermedad de Alzheimer, llamado galantamina. Entonces... ¿por qué enfocarnos en Amaryllidaceae y Alzheimer? Porque además del alcaloide galantamina hay otros alcaloides que produce la familia que pueden tener actividad biológica igual o superior a la galantamina. Así, con este proyecto quiero ser un poco más ambiciosa y evaluar si las Amaryllidaceae que crecen en el Tolima tienen un potencial para el tratamiento no solo paliativo sino también modulador de la enfermedad de Alzheimer.

\section{Además del tratamiento de la enfermedad de Alzheimer, ¿en qué otras enfermedades considera que tendría un impacto positivo el tratamiento con alcaloides de Amaryllidaceae?}

Hay dos tipos de enfermedades con alto índice de mortalidad en las cuales los alcaloides de Amaryllidaceae tienen un importante valor farmacológico: la enfermedad de Alzheimer y el cáncer. En este momento soy coinvestigadora de un proyecto con la Universidad de Antioquia, liderado por el doctor Edison Javier Osorio Durango, en el

${ }^{2}$ U.S. Food and Drug Administration 
que estamos evaluando la actividad antitumoral de diferentes Amaryllidaceae colectadas en el país, incluido Tolima, con varios tipos de cáncer como mama, cuello uterino, estómago, próstata y pulmón. Aunque aún no tenemos resultados experimentales, hay una importante evidencia científica que señala el potencial farmacológico de la familia Amaryllidaceae y cáncer.

En el departamento del Tolima existen dos especies endémicas y tres nativas que hacen parte de la familia Amaryllidaceae, ¿considera que esta es una variable importante para realizar más investigaciones de este tipo?

Sí, es muy importante porque primero son especies que no han sido estudiadas tanto química como biológicamente. Por lo tanto, estudios de este tipo son de vital importancia puesto que al estudiarlas potencia su interés químico y biológico haciendo inminente su conservación tanto a nivel regional como nacional.

Una de estas plantas crece en la Universidad de Ibagué; su nombre científico es Zephyranthes puertoricensis y es una de las protagonistas del proyecto, dado que hasta la fecha no se han reportado estudios químicos ni de su actividad biológica. Ella también hace parte del estudio de cáncer que se está llevando a cabo con la Universidad de Antioquia y esperamos encontrar en esta planta, y en las demás que se colecten en el Tolima, un potencial promisorio para algún tipo de enfermedad.

Figura 2. Zephyranthes puertoricensis en la Universidad de Ibagué
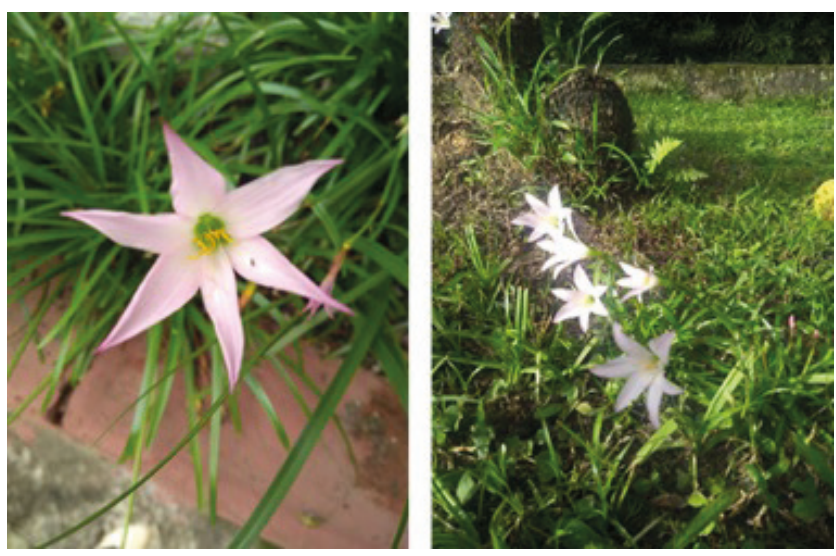

Fuente: Natalie Cortés 
El departamento del Tolima, por su ubicación geográfica, es rico en flora y fauna. En él se encuentran especies nativas y endémicas, sin embargo, muchas de estas han sido poco estudiadas. Se espera que el desarrollo del proyecto Amaryllidaceae del Tolima: fuente inexplorada de alcaloides para el tratamiento de la enfermedad de Alzheimer brinde información de la familia Amaryllidaceae a la comunidad científica del departamento, la región y el mundo, otorgando conocimiento de su importancia ecológica para los ecosistemas y contribuyendo a la preservación y conservación de la familia en el departamento.

\section{Referencias}

Alzate, F., Lesmes, M., Cortés, N., Varela, S., \& Osorio, E. (2019). Sinopsis de la familia Amaryllidaceae en Colombia. Biota Colombiana, 20(1), 2-20. https://doi.org/10.21068/c2019.v20n01a01

Cabezas, F., Pigni, N., Bastida, J., Codina, C., \& Viladomat, F. (2013). Analisis del contenido alcaloidico de caliphruria subedentata baker (amaryllidaceae) por el metodo CG-EM. Revista Latinoamericana de Quimica, 41(1), 68-73.

Cortés, N., Posada-Duque, R., Cardona-Gómez, G. P., Bastida, J., \& Osorio, E. (2020). Amaryllidaceae alkaloids and neuronal cell protection. In Pathology (pp. 135-144). https://doi.org/10.1016/b978-012-815972-9.00013-5

Meerow, A. W., \& Snijman, D. A. (1998). Amaryllidaceae. In Flowering Plants · Monocotyledons (pp. 83110). https://doi.org/10.1007/978-3-662-03533-7_11 